\title{
The Management of a Comprehensive Cancer Center during the First Six Months of the COVID-19 Pandemic in the South of France: Lessons from the Paoli-Calmettes Institute's Experience
} \author{
Marc Giovannini ${ }^{8}$, Gilles Houvenaeghel ${ }^{3,9}$, Olivier Turrini ${ }^{3,10}$, Christian Chabannon ${ }^{2,3,11}$, \\ Gilles Piana ${ }^{12}$, Isabelle Brenot-Rossi ${ }^{13}$, Agnès Tallet ${ }^{14}$, Anthony Gonçalves ${ }^{2,3,15}$, \\ Aude Charbonnier ${ }^{1}$, Norbert Vey ${ }^{1,2,3}$, Sabrina Grossi ${ }^{16}$, Patrice Viens ${ }^{3,15,16,17, \dagger}$ \\ ${ }^{1}$ Department of Hematology, Institut Paoli-Calmettes, Marseille, France \\ ${ }^{2}$ Centre de Recherche en Cancerologie de Marseille (CRCM), Inserm, CNRS, Marseille, France \\ ${ }^{3}$ Aix-Marseille University (AMU), Marseille, France \\ ${ }^{4}$ Infectious Diseases Committee, Institut Paoli-Calmettes, Marseille, France \\ ${ }^{5}$ Department of Anesthesiology and Critical Care, Institut Paoli-Calmettes, Marseille, France \\ ${ }^{6}$ Department of Cancer Ambulatory Care, Institut Paoli-Calmettes, Marseille, France \\ ${ }^{7}$ Pharmacy, Institut Paoli-Calmettes, Marseille, France \\ ${ }^{8}$ Medical and Surgical Endoscopy Cancer Unit, Institut Paoli-Calmettes, Marseille, France \\ ${ }^{9}$ Department of Cancer Surgery 2, Institut Paoli-Calmettes, Marseille, France \\ ${ }^{10}$ Department of Cancer Surgery 1, Institut Paoli-Calmettes, Marseille, France \\ ${ }^{11}$ Cellular Therapy Platform, Institut Paoli-Calmettes, Marseille, France \\ ${ }^{12}$ Department of Cancer Imagery, Institut Paoli-Calmettes, Marseille, France \\ ${ }^{13}$ Department of Nuclear Medicine, Institut Paoli-Calmettes, Marseille, France \\ ${ }^{14}$ Department of Radiotherapy, Institut Paoli-Calmettes, Marseille, France \\ ${ }^{15}$ Department of Medical Oncology, Institut Paoli-Calmettes, Marseille, France \\ ${ }^{16}$ General Direction, Institut Paoli-Calmettes, Marseille, France \\ ${ }^{17}$ Sport Cancer Laboratory EA4670, Luminy Campus, Aix-Marseille University, Marseille
}

Didier Blaise ${ }^{1,2,3, *, t,(\mathbb{D}, \text { Pierre Berger }}{ }^{4}$, Djamel Mokart ${ }^{5}$, Jacques Camerlo ${ }^{6}$, Emmanuelle Fougereau ${ }^{7}$,

\section{ARTICLE INFO}

Article History

Received 16 June 2021

Accepted 05 September 2021

Keywords

COVID-19

pandemic

hospital management

real-world experience

lessons learned

\begin{abstract}
During the COVID-19 pandemic, it was rapidly established that cancer patients have an increased risk of developing severe forms of the 2019 coronavirus disease (COVID-19) due to a backlog of cancer diagnostics and immunosuppressive treatments. Cancer centers had to quickly adapt to continue cancer therapies despite the high infection risks and major disruptions in the French healthcare system. We described and analyzed the impact of the pandemic in our institution: management adjustments, COVID-19 infection rates in patients and staff, and impacts on clinical activities and finances during the first wave of the pandemic from March to September 2020. We also compared the results to the clinical activity data from preceding periods. A crisis unit was rapidly created that met 27 times over 66 days, generating numerous changes in hospital protocol. While our area was devastated by the pandemic, the infection rate of our staff and patients remained low (less than $1.5 \%$ of all employees). However, the lockdown period was accompanied with a reduction of most clinical activities, leading to decreases of $43 \%$, $36 \%, 36 \%, 1 \%$, and $10 \%$ in surgery, endoscopy, radiotherapy, and in- and out-patient chemotherapy sessions, respectively, with substantial financial loss. Our report highlights the need for the rapid creation, implementation, and adaptation of new protocols during a pandemic's evolution to prevent disease transmission. Lessons from this situation should provide motivation to better prepare for/limit the dismantling of cancer therapies that can dramatically impact patient care and have deleterious consequences on an institution's financial situation.
\end{abstract}

(c) 2021 International Academy for Clinical Hematology. Publishing services by Atlantis Press International B.V. This is an open access article distributed under the CC BY-NC 4.0 license (http://creativecommons.org/licenses/by-nc/4.0/).

\section{INTRODUCTION}

At the end of 2019, a novel infectious disease of likely zoonotic origin related to the severe acute respiratory syndrome (SARS)

Corresponding author.Email: blaised@ipc.unicancer.fr

${ }^{\top} \mathrm{DB}$ and PV contributed equally to this paper.

Peer review under responsibility of the International Academy for Clinical Hematology coronavirus-2 (SARS-CoV-2), emerged in China and quickly spread across the globe [1,2]. Named the coronavirus disease 2019 (COVID-19) it is characterized by both a high human-tohuman transmission rate and the subsequent development of SARS [3]. The number of infected individuals and related deaths increased daily thereafter, approaching 176 and 3.8 million, respectively, as of June 14, 2021 in over 191 countries (https:// coronavirus.jhu.edu/). 
Mortality was rapidly found to be higher in patients with increased age, preexisting comorbidities, and chronic diseases [4]. In particular, initial and following reports indicated that cancer and immunocompromised patients have a higher risk of infection and present a higher incidence of severe events [4-13].

Due to an incubation period of 2-14 days [14], the World Health Organization (WHO) has recommended rigorous hygiene measures, isolation of infected individuals, and social distancing to limit cases and subsequent severe events.

Experiences on the care of cancer patients in general oncology units during to the pandemic have been widely reported [15], aiding greatly in the rapid issuing of general recommendations (https://cancerletter.com/conversation-with-the-cancerletter/20200313_3/) [16-19]. However, a large number of cancer patients are currently treated in specialized institutions that focus on providing state-of-the-art treatment. These centers congregate numerous patients who are at high risk of severe complications [20] and, consequently, had to adapt even more rapidly than other more generalized cancer centers to provide the greatest possible safety to patients, while simultaneously preserving their chances of successful cancer treatment [21].

After evaluating our patient typology, regional health authorities assigned to our hospital the primary mission of remaining COVID-19-free for as long as possible, while continuing anticancer treatments (details in online-only supplemental data).

Therefore, we decided to report the experience of the PaoliCalmettes Institute (IPC, details in online-only supplemental data), a major French comprehensive cancer center in the south of France, from the first 6 months of the pandemic with the goal of augmenting the global literature and subsequently improving future responses to similar crises.

In our study, we aimed to critically analyze the protocol adjustments implemented to fulfill our primary mission, as well as their immediate and secondary impacts on patient treatment and hospital activity.

\section{MATERIALS AND METHODS}

The IPC is part of the UNICANCER French Cancer Center Federation and certified by the Organization of European Cancer Institutes (OECI ID: 100). Every year more than 11,000 new patients, totaling 45,000 when added to existing patients, are treated at the IPC with more than 100,000 consultations. The inpatient hospital capacity is 273 beds, 80 of which are equipped with laminar airflow for the Hematology Department and 20 for intensive care. Our hematology-oncology activity includes one of the most active transplant programs in Europe (125-150 allogeneic and 100-120 autologous hematopoietic stem cell transplants per year). IPC uses more than 1700 persons of which over 230 are permanent medical doctors. The majority of patients reside within five of the six departments of the Provence-Alpes-Cote d'Azur (PACA) region and the two departments of Corsica, whose combined total population is approximately 4.3 million inhabitants.

The first COVID-19 cases were reported in China, USA, France, and Italy on $31 / 12 / 19,23 / 01 / 20,24 / 01 / 20$, and $31 / 01 / 20$, respectively (https://coronavirus.jhu.edu/data/cumulative-cases), while the first 18 cases in the PACA region were officially recorded on 04/03/20. By August 31st, 2020 -the end of our analysis- a total of 35,286 cases and 1289 deaths had been documented in hospitals and nursing homes (data from PACA ARS: https://www. paca.ars.sante.fr/liste-communiques-presse). Considering both the initial reports on the characteristics of the pandemic in other countries and, notably, the needs of long-term ICU resources for SARS development, French health authorities selected hospitals throughout the country to act as the front-line in diagnosing and treating suspected and infected patients. Several different lines of hospitals were defined, to be activated as needed. In the PACA area, the first and second lines were progressively activated in March. Our institution was not a part of these initial lines. However, we were asked to prepare our ICU facility for the transfer of COVID-negative patients requiring intensive care from other overwhelmed hospitals. Although we felt that these missions might be somewhat contradictory and quite difficult, we were fully committed to them both.

Once the health authorities issued their general recommendations, and it became likely that our area would be impacted by COVID-19, we activated and adapted our crisis plan accordingly. Since activation occurred before our hospital had been affected by the pandemic, we had the advantage of having some time to prepare, though the magnitude of the predicted impact was unknown. This pushed us to prepare for the worst-case scenario, i.e., regional health resources flooded by large numbers of patients, as it was ongoing in Northern Italy and the north-east of France at that time.

At the national level, the preventive measures enacted to limit transmission progressed from the promotion of good hygiene procedures and social distancing in February to the national lockdown on March 17th (D11 of this analysis). On March 24th (D18), the National State of Health Emergency (NSHE) was declared alongside the implementation of specific measures in healthcare institutions. Lockdown measures were amended progressively from May 11th (D66) until the NSHE was revoked on July 11th (D127).

Our study's data collection period began on March 6th, 2020 (D0), the date the crisis unit was created, and included the six subsequent months. This period allowed us to investigate different and successive situations: the start of the pandemic, the strict lockdown and its progressive alleviation, and the eventual return to "normal" life despite the continued transmission of the virus.

We report different quantitative values:

The recorded daily number of COVID-19 reverse transcriptase polymerase chain reaction (RT-PCR) tests performed in the IPC for both patients and staff until July 11th (D127), the end of the NSHE. After this date, the availability of testing in private laboratories disrupted data collection. Detection policy gradually changed over this period due to several new developments. Initially, only clinical evaluations and epidemiology data (travel history; contact with positive cases) were available to us. Then, RT-PCR results became available at the reference center in Marseille, but with limited numbers and a delay of more than $24 \mathrm{~h}$ for results. However, the potential for false negatives in $30-40 \%$ of cases was a cause for concern regarding our attempt at creating a safe and secure strategy for our hospital. Furthermore, we were also rapidly faced 
with false positive results that took several days for correction. Yet, despite these uncertainties, we enlarged the indications for RT-PCR in both the patient and staff populations as soon as testing availability increased. In order to improve diagnostic accuracy in patients, we progressively carried out pulmonary CT-scans in addition to RT-PCR due to the reportedly good correlation with PCR and rapid availability. However, in a non-negligible number of cases, the results from both tests were contradictory and made for a highly challenging situation. A special committee was created to decide on these cases. For hospital staff, RT-PCR was initially carried out only in symptomatic individuals or people in contact with diagnosed COVID-19 individuals, and progressively increased according to less stringent criteria.

- Clinical activity:

- Hospital bed occupancy was used to represent clinical activity. The number of occupied beds per department was recorded daily.

- Several other activities were also recorded including the number of consultations; chemotherapy courses; visits to medical outpatient department (OPD); and surgical, radiotherapy, chemotherapy, transplantations, and medical or nuclear medicine imaging procedures.

- Monthly hospital income as reimbursed by national health insurance according to reported clinical activity.

- Data collection began at week 12 (start of national lockdown) and continued for 6 months. Bed occupancy, activity, and financial income from each week of the study period were respectively represented as a ratio with the total number of beds, the median number of procedures, or the median revenue assessed during the first 8 weeks of 2020, prior to the start of the pandemic in the region.

\section{RESULTS}

A crisis unit (Table 1) was established after an initial preparatory meeting (D0). This unit consisted of the General Director, Deputy Director, two representatives from the medical council, the chair of the Infectious Diseases Committee, and the Nurse and Human Resources management. The unit sequentially invited representatives from different departments to be apprised of information from the entire hospital and communicate decisions issued by the unit to each department. From the first meeting until the end of the strict lockdown period (66 days), 27 meetings were held. During these meetings, numerous specific consultations were scheduled with numerous hospital stakeholders. Decisions were regularly publicized to all staff by the director or deputy director via the IPC's intranet as well as specific newsletters disseminated by mail.

During each of the crisis unit's meetings, members reviewed the number of positive and suspected cases among staff and patients as well as the status of critical material stocks, such as personal protective equipment (PPE) and anesthetics. Additionally, three primary areas were defined and regularly assessed, each of them having obvious impacts on the others: protection of patients and staff, detection and management of suspected or diagnosed COVID-19 individuals, and modulation of clinical activity.

\subsection{Implementation of Safety Measures for Patients and Staff}

- Mask protocol was modified according to anticipated mask shortages, despite an early increase in orders, the projected duration of the outbreak, and symptomatology of individuals. Thus, only symptomatic individuals were initially asked to wear a mask and when resources became sufficient this policy was later expanded to include first all staff and then all patients coming to the hospital (Table 1).

- On average, more than 400 patients arrive each day for consultations. To reduce this number, routine long-term consultations were postponed for several months whenever possible and accompanying persons were limited to those patients requiring immediate assistance. In less than 1 week the number of consultations dropped by more than $40 \%$ (Figure 1). At the same time, teleconsultations were widely offered. The modification and rescheduling of consultations were carried out by the patients on the IPC app. With the same pace, $60 \%$ of the remaining consultations were switched to online procedures (Figure 1).

- Entering the physical site was limited to a single entrance per building. At each checkpoint, the clinical symptoms of every individual were investigated.

- No direct admittance to hospitalization units was allowed.

- Outpatients presenting COVID-19-like symptoms were asked to first call the regional COVID-19 hotline that would further review the situation and refer patients to their general practitioner (GP) or to COVID-19 units. If patients without COVID-19 symptoms required unexpected care they were asked to call the IPC's newly created hotline. After an oral debriefing of the situation, the patients were either advised to stay home under GP care or invited to present themselves to the triage unit, where further examinations were performed.

- All patients scheduled for hospitalization were called on the day before admittance. When COVID-19 symptoms were reported, the patient was asked to consult their GP. Patients were asked again concerning symptoms upon arrival to the IPC entrance, after which they could then be directed to the triage unit for further investigations.

A hotline operated by a recently retired, volunteer Medical Doctor (MD) was established on March 27th to answer questions from IPC patients, family members, and GPs on instructions for cancer patient care in the context of the pandemic. The phone number was disseminated via the IPC's patient app as well as posted on the IPC's website. Starting the first day and over the following period of 50 operating days ( 5 days per week; 8:00 am to 6:00 pm), the hotline received 748 calls (median 12; range 2-64). The hotline was discontinued after 84 days due to a decrease in incoming calls.

As of the end of the state of health emergency (115 days), 753 and 1184 RT-PCR tests were conducted in patients and staff, respectively (Figure 2 and online-only supplemental data). Two patients and 32 staff members were found to be RT-PCR positive. COVID-19 may have contributed to the death of one patient with advanced 
Table 1 Chronologic minutes of main institution-wide decisions made by the crisis unit

\begin{tabular}{|c|c|c|}
\hline & February & $\begin{array}{l}\text { Increase in the institution-wide supply orders for masks, protective garments, anesthetic, and anti-infective drugs in case } \\
\text { of possible outbreak }\end{array}$ \\
\hline Day-7 & Feb 28th & $\begin{array}{l}\text { Information meeting between the institution's general leadership, medical staff delegates, and support department } \\
\text { representatives }\end{array}$ \\
\hline \multirow[t]{3}{*}{ Day+0 } & March 6th & First crisis unit meeting \\
\hline & & Definition of suspicion of COVID-19 based on symptoms and travel history \\
\hline & & Implementation of a care policy until result of RT-PCR \\
\hline \multirow[t]{5}{*}{ Day+3 } & March 9th & Restriction of visitors' visits \\
\hline & & Switch from in-person to teleconsultations \\
\hline & & Implementation of new directives for mask use: \\
\hline & & Surgical masks were distributed and asked to be worn by front desk clerks, symptomatic patients, and staff \\
\hline & & $\begin{array}{l}\text { FFP2/N95 masks were restricted to nursing and medical staff during surgery or at-risk care of suspected patients } \\
\text { (toilets, endoscopy, intubation...) }\end{array}$ \\
\hline \multirow[t]{3}{*}{ Day+6 } & March 12th & Banning of visits from external professionals and in-person internal seminars and lessons \\
\hline & & Promotion and implementation of a web-based solution for internal meetings \\
\hline & & Limitation of non-essential external business meetings \\
\hline \multirow[t]{3}{*}{ Day+7 } & March 13th & Every department was asked to establish a reduced activity plan with the hypothesis of $25 \%$ of staff unavailable \\
\hline & & Intensification of general cleaning of at-risk areas (elevators, doorknobs...) \\
\hline & & Implementation of adapted policies for suspected or positive COVID-19 deceased patients edited by national agency \\
\hline Day+8 & March 14th & Surgical masks to be worn by all nursing staff \\
\hline \multirow[t]{7}{*}{ Day +10} & March 16th & $\begin{array}{l}\text { Closing of hospital to all visitors except for staff and patients; Closing-down of staff restaurant facilities replaced by } \\
\text { 'to-go' meal options }\end{array}$ \\
\hline & & Preparation of ICU resources to care for transferred patients from other hospitals \\
\hline & & $\begin{array}{l}\text { Deprogramming of elective surgery to free up ventilators from surgery theater and to strengthen operational bed } \\
\text { capacities in ICU }\end{array}$ \\
\hline & & Determination of specific paths of circulation for expected COVID-19 patients inside the hospital \\
\hline & & Implementation of teleworking policy for non-care staff \\
\hline & & $\begin{array}{l}\text { Activation of medical backup from recently retired medical staff, relocation of administrative and research staff to } \\
\text { transversal support functions }\end{array}$ \\
\hline & & Suspension of all holidays for MDs \\
\hline Day+11 & March 17th & Activation of phone Hotline for patient guidance \\
\hline Day+12 & March 18th & Freezing clinical trial protocol inclusions \\
\hline \multirow[t]{4}{*}{ Day +14} & March 20th & Mailing out prescriptions and instructions to outpatients \\
\hline & & Entrance to hospital restricted to a single-entry door with COVID-19 monitoring staff \\
\hline & & Tutorials for strict mask use and gowns posted on institutional intranet and conveyed by individual e-mails \\
\hline & & Modifications of waiting area to limit contacts \\
\hline Day+16 & March 22nd & Surgical masks to be worn by all individuals within the hospital \\
\hline Day+17 & March 23rd & $\begin{array}{l}\text { Creation of a new patient transit facility with dedicated staff to check and accordingly sort patients prior to any } \\
\text { hospitalization }\end{array}$ \\
\hline Day +21 & March 27th & Transit unit was converted into a short-term hospitalization unit to allow for the time necessary for tests results \\
\hline \multirow[t]{2}{*}{ Day+29 } & April 4th & $\begin{array}{l}\text { Modification of the sorting algorithm according to the COVID-19 status (negative; suspected; positive) based on } \\
\text { symptoms, RT-PCR, and pulmonary CT-scan evaluation }\end{array}$ \\
\hline & & Survey of the use and efficiency of the procedure by a multi-disciplinary COVID-19 committee \\
\hline Day +32 & April 7th & First meeting of a specific COVID-19 ethical committee to support staff in difficult situations or decisions \\
\hline Day +40 & April 15th & Resumption of surgical activity \\
\hline
\end{tabular}

disease while one employee was hospitalized for several weeks in an ICU but eventually recovered.

Our standard weekly capacity is 1877 beds. Figure 3a shows the percentage of occupied beds per department and per week. While the median occupation ratio normally surpassed 95\%, a significant decrease was seen after week 12 . During the national lockdown period, a total of 2673 beds (18\%) remained unoccupied, equivalent to 1.4 weeks of full capacity over an 8 -week period. This decrease was mainly seen in the three surgery-related departments, accounting for $71 \%$ of unoccupied beds and $58 \%$ of unused hospitalization capacity during this period. At the same time, the number of beds for unscheduled stays was increased from seven (8:00 am to 6:00 pm, 5 days/week) to 17 beds ( $24 \mathrm{~h} /$ day, 7 days/week, details in online-only supplemental data) to take into account the duration of COVID-19 diagnostic procedures. Medical outpatient activity is depicted in Figure 3b, with a $24 \%$ decrease mainly seen in relation to fewer screening visits (details in online-only supplemental data).

The main parameters of clinical activity are presented in Figures $4 \mathrm{a}$ and $4 \mathrm{~b}$. For every recorded activity, we observed a major decrease during the lockdown period, which was the most pronounced in surgery. Over these 8 weeks, clinical activity was reduced by $43 \%$, $36 \%, 36 \%$, $1 \%$, and $10 \%$ for surgery, endoscopy, radiotherapy, and inpatient and outpatient chemotherapy sessions, respectively. During the second period, post-lockdown, some clinical activity increased but was still less than the previously expected: $-20 \%, 0 \%$, $-13 \%,+5 \%$, and $-8 \%$, respectively. During the third period, the 2 months following the lifting of the state of health emergency, the clinical activity remained lower with only $75 \%, 87 \%, 85 \%, 104 \%$, and $91 \%$ of expected activity in the different departments, respectively. Only inpatient chemotherapy remained within or above the expected range. 


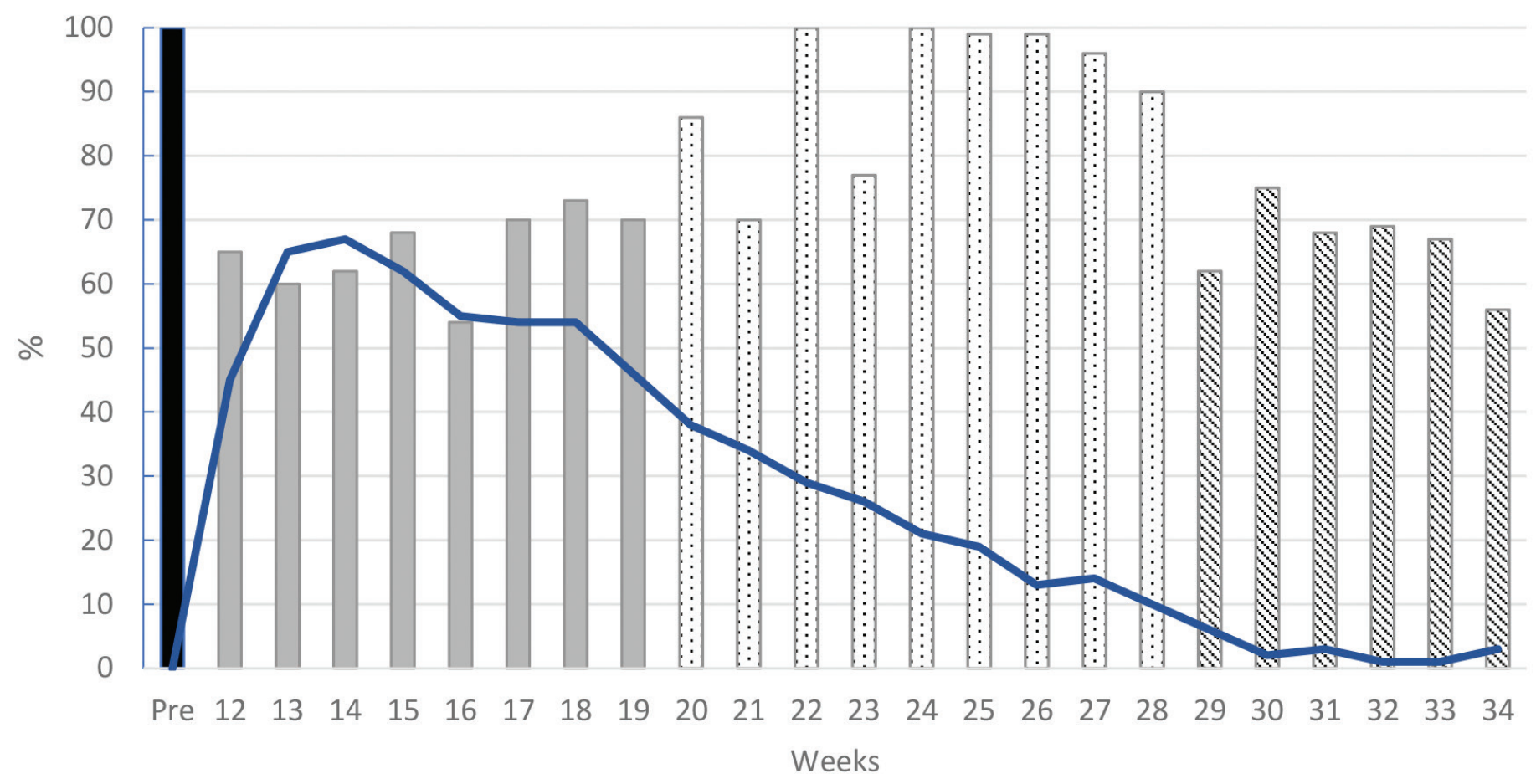

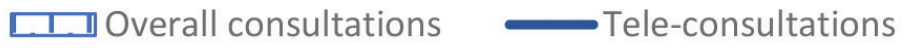

Figure 1 Consultation activity. Data are presented as a ratio between the weekly reported activity and the median activity of the first 2 months preceding the pandemic in the year 2020 (illustrated as $100 \%$ in bar pre).

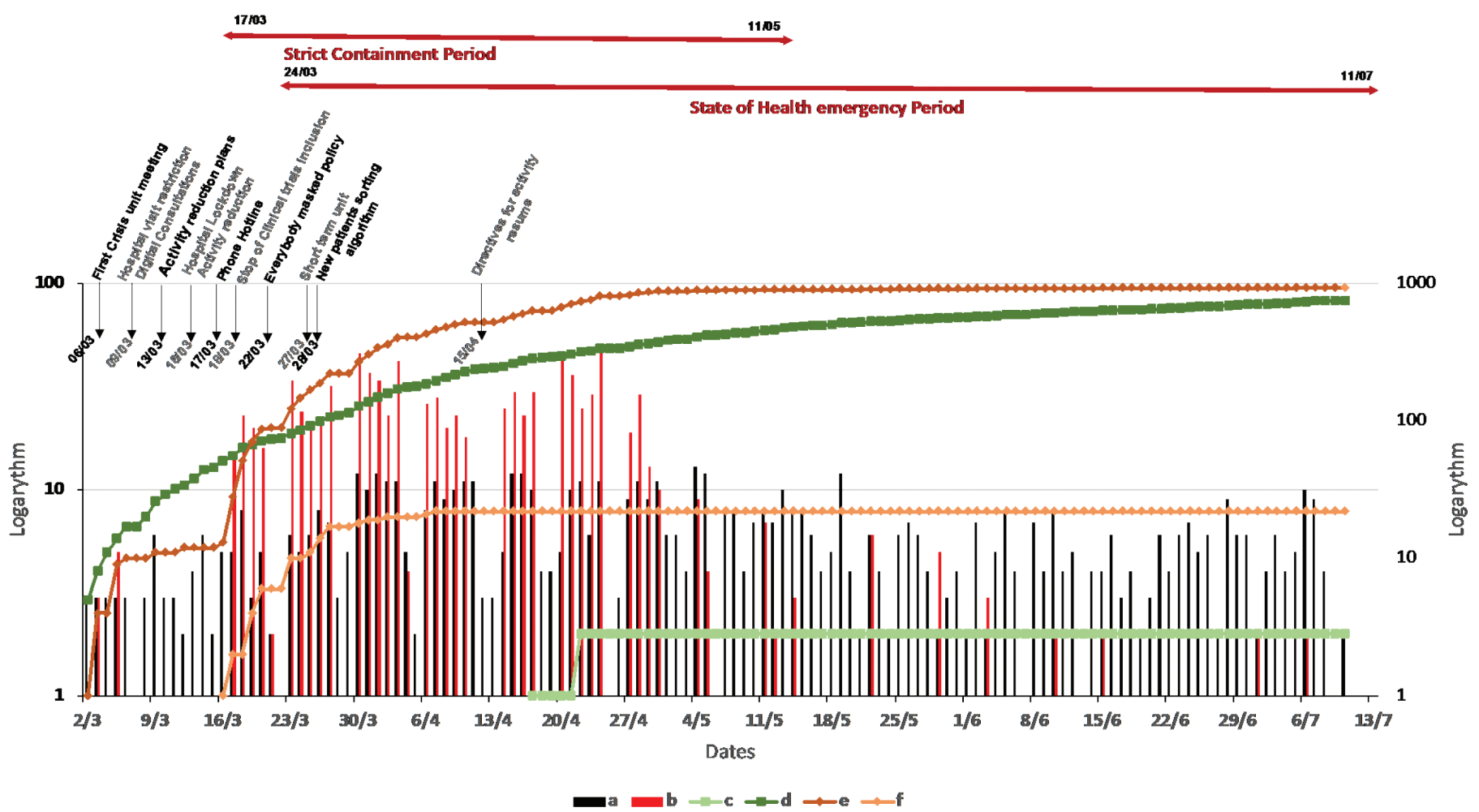

Figure 2 Daily evaluation of polymerase chain reaction tests in staff and patients Logarithmic representation. (a) Daily tests in patients (left axis). (b) Daily tests in staff (left axis). (c) Cumulative positive tests in patients (right axis). (d) Cumulative tests in patients (right axis). (e) Cumulative tests in staff (right axis). (f) Cumulative positive tests in staff (right axis). 
a

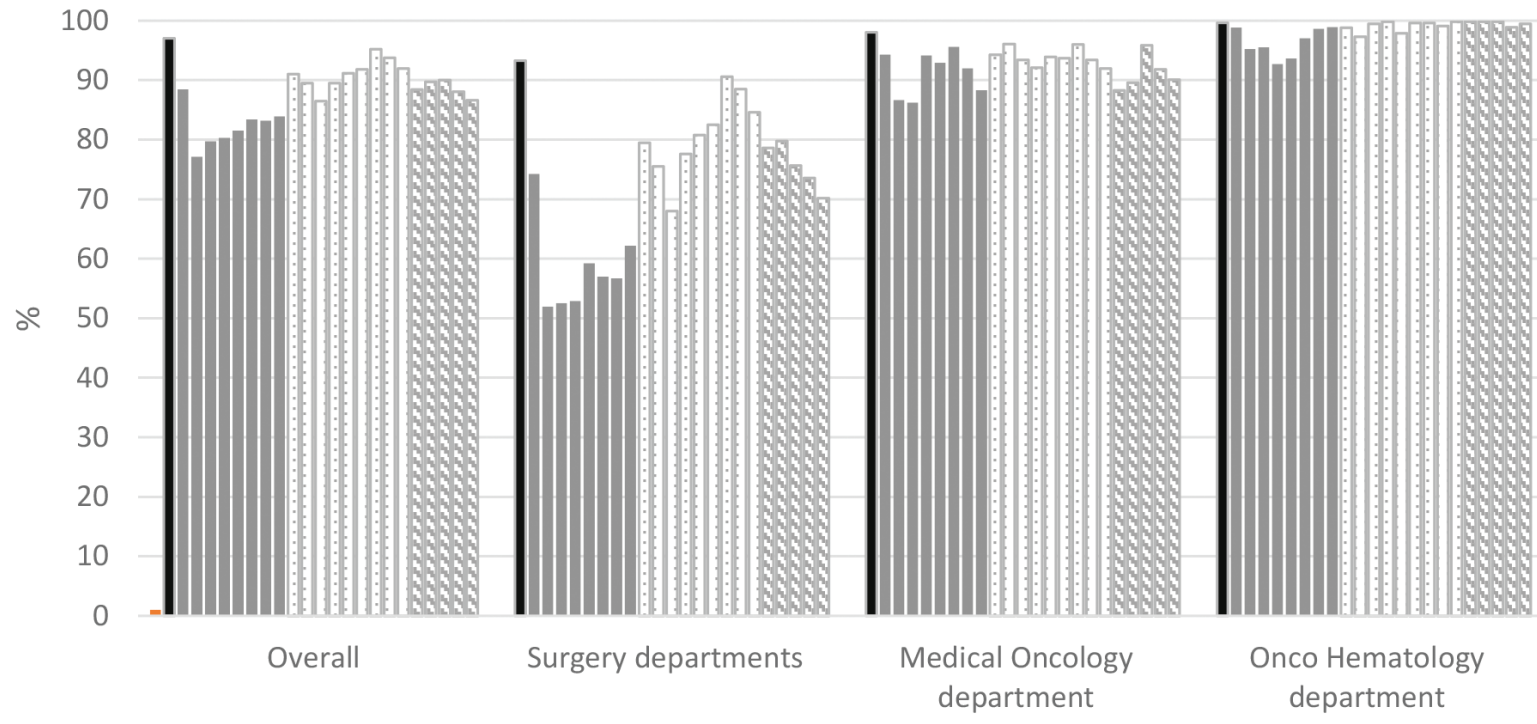

Weeks

b

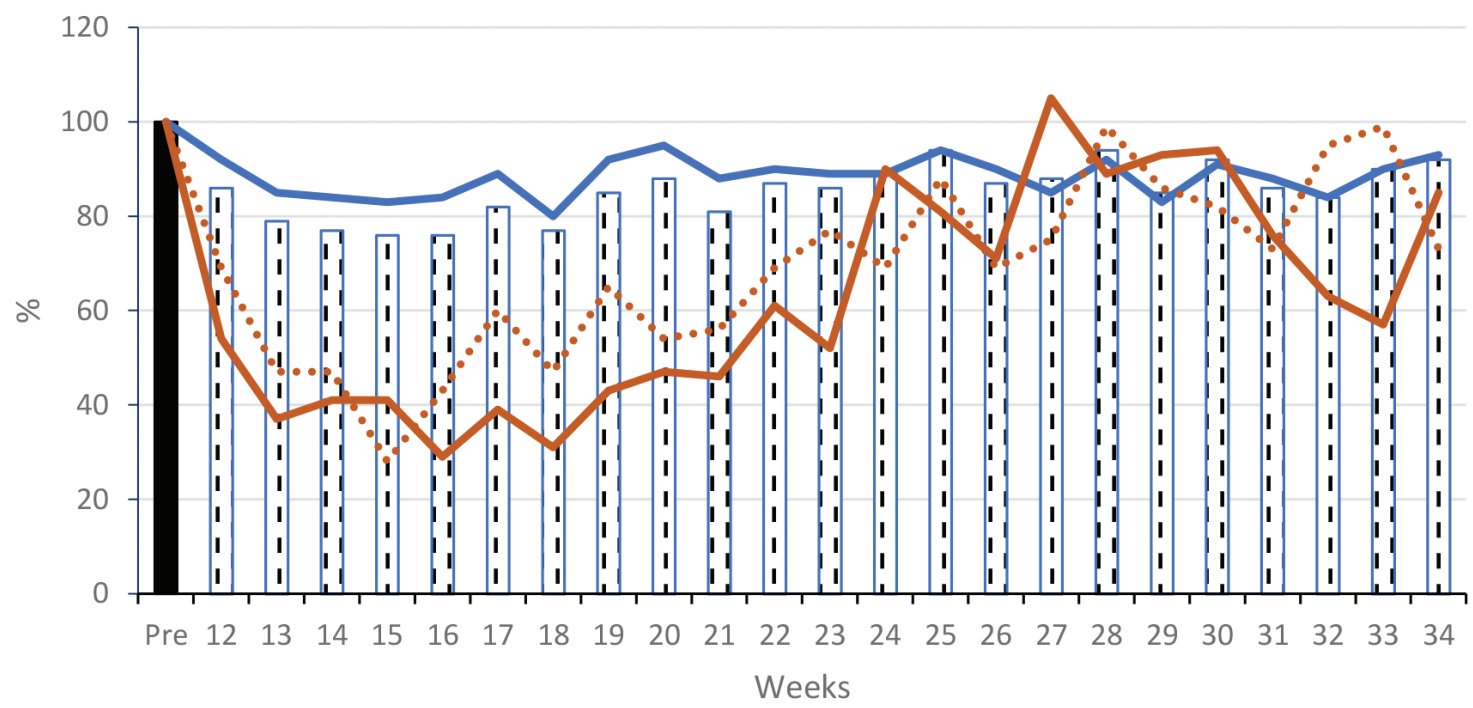

[.I Total outpatient department sessions Chemotherapy Sessions

..... Transplant Related sessions $\quad$ Clinical or biological monitoring visits

Figure 3 Weekly inpatient bed occupancy and outpatient activity. (a) Inpatient bed occupancy. (b) Medical outpatient department activity. All activities are presented as a ratio between the weekly reported activity and the median activity of the first 2 months preceding the pandemic in 2020 (illustrated as $100 \%$ in bar pre).

Medical imaging via magnetic resonance imaging (MRI), computerized tomography (CT), or positron emission tomography (PET) scans followed the same pattern (Figure 4c). The initial activity for CT, PET, and MRI scans decreased by $16 \%, 35 \%$, and $54 \%$ during the first period; varied by $+16 \%,-11 \%$, and $+1 \%$ during the second; and decreased by $2 \%, 29 \%$, and $30 \%$ during the third, respectively. The number of CT-scans performed increased when pulmonary CT-scans became part of the diagnostic algorithm.

Hematopoietic stem cell transplantation (HSCT) and cell-based immunotherapies, such as chimeric antigen receptor (CAR) T-cell treatments, were also impacted by the pandemic. Due to the recommendations from national and international scientific communities [22] as well as the practical realities, we were unable to perform any allogeneic HSCT during a 5-week period after the start of the lockdown (details in online-only supplemental data). Some transplants were cancelled while others were delayed. The decrease in activity was mainly seen in allogeneic transplantation $(-61 \%$ when compared to the activity of the same period the previous year: data not shown) while autologous and CAR-T cell therapies $(+2 \%)$, for which cellular products were already harvested, were largely unaffected. Allogeneic transplantation activity was partly restored during the subsequent periods, though some patients never received their transplant due to disease progression or worsening health status. 
a

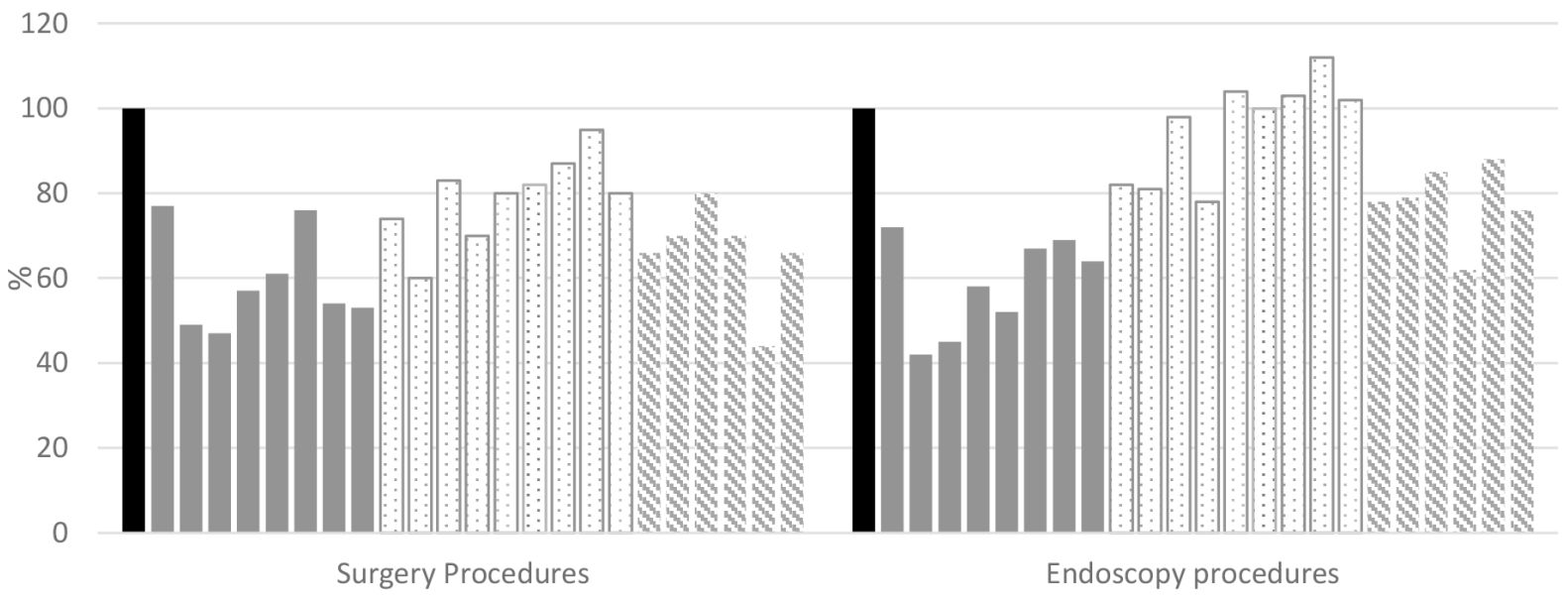

Weeks

b
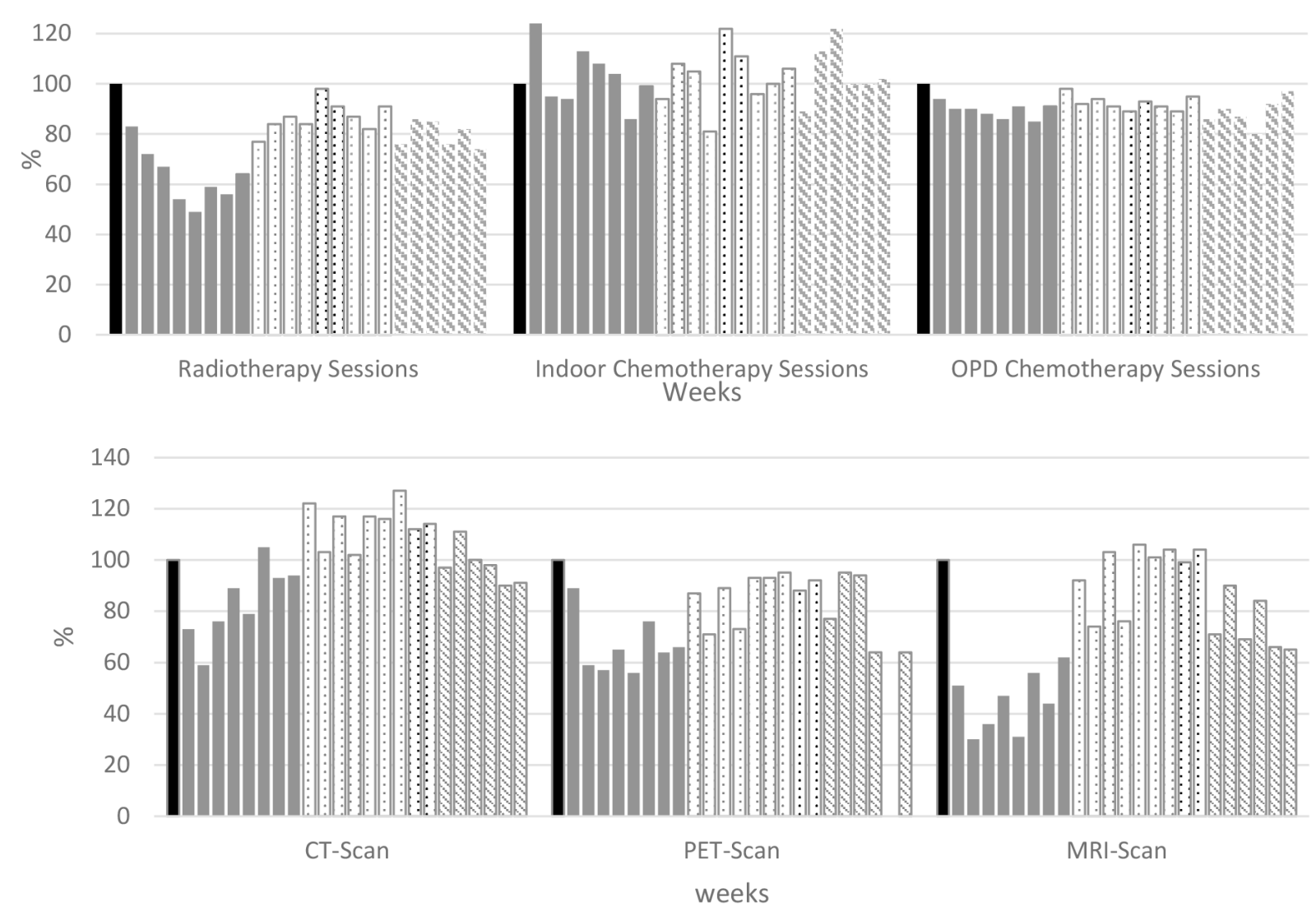

Figure 4 Treatment and imaging activities. (a) Surgery and endoscopy procedures. (b) Radiotherapy and chemotherapy sessions. (c) Imaging procedures. All activities are presented as a ratio between the weekly reported activity and the median activity of the first 2 months preceding the pandemic in 2020 (illustrated as $100 \%$ in bar pre).

\subsection{Financial Appraisal}

The first 2 months of 2020 reflected the same financial growth observed during 2019 for the IPC (data not shown) (Figure 5). Compared to this increase, the subsequent months saw a major financial decrease during the lockdown period (reaching median of $-13 \%$ for the months of March to May 2020), which was not fully restored during the next 3 months.

\section{DISCUSSION}

As a cancer center, we were especially committed to keeping our institute free from COVID-19 due to the anticipated risks of infection in immunocompromised cancer patients. From this perspective, the actions we took can be considered to have been successful. Less than $1.5 \%$ of our staff was infected, and most of these from transmission outside the hospital, while all patients 


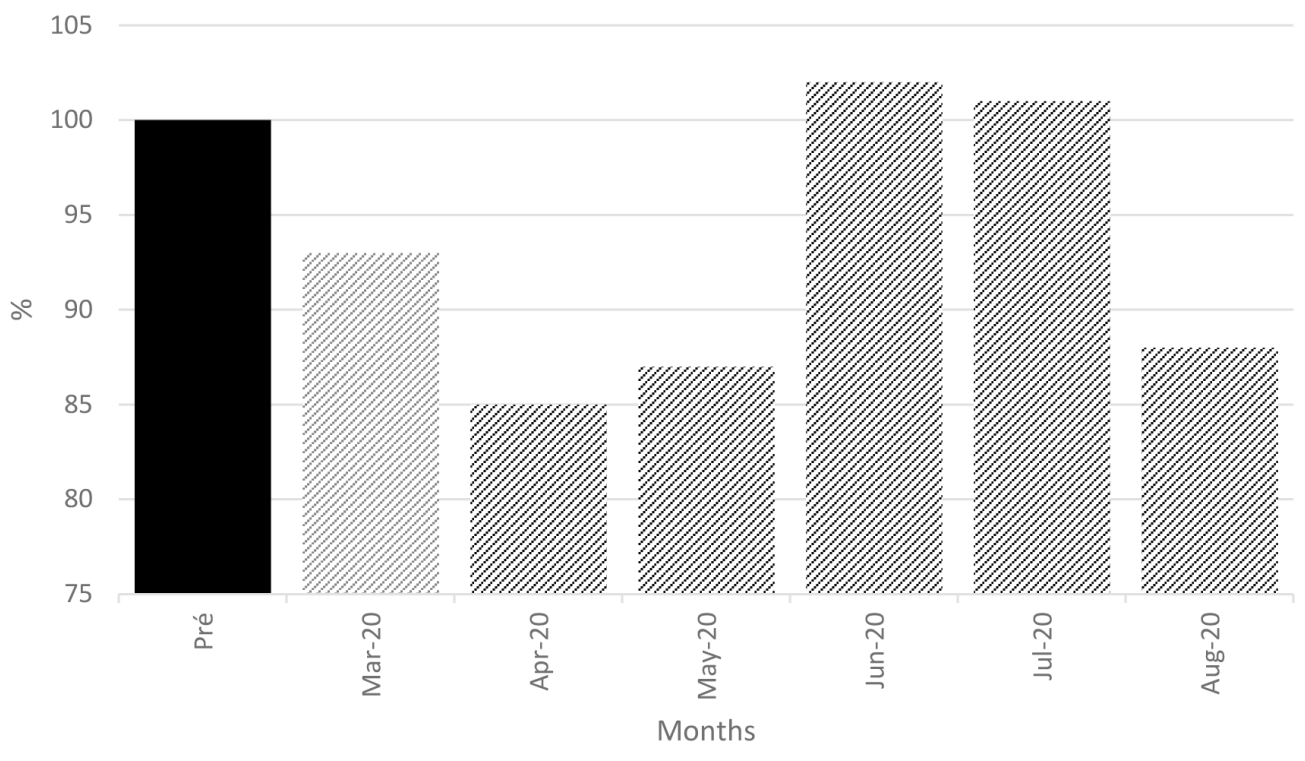

Figure 5 Institution's monthly revenue. Ratio between the weekly reported revenue and the median revenue of the first 2 months preceding the pandemic in the year 2020 (illustrated as 100\% in bar pre).

considered to be COVID-19 positive were referred to dedicated facilities. In fact, we efficiently promoted attentiveness to social distancing, hand washing, and masking [23,24]. Although these measures appear simple, their implementation was challenging for our healthcare facility comprising numerous buildings and accommodating 1700 employees and an estimated number of greater than 6000 patients and visitors each month.

That being said, our lack of preparation for this crisis was obvious. Various scenarios had been previously developed in our crisis plans, but were based on a sudden major accident or attack scenario, with little impact on the center's human resources. In those instances, we had anticipated that, after the initial hours or days, we could rapidly receive aid from other regional or national structures. The reality was totally different.

Initially, the disease was known about for several weeks before reaching France, with conflicting and perturbing "expert opinions" issued both in the scientific literature and, more widely and rapidly, in the media. Additionally, awareness of what occurred in previously affected areas prompted us to rapidly prepare for a massive and long-lasting "war scenario" that would significantly impact our current organization. This rationale was emphasized by national general recommendations that could not reflect the local reality. Thus, we had to address several contradictory objectives at once: protecting patients and staff from a highly infectious and potentially devastating virus, being ready to support other healthcare institutions, while maintaining anticancer activity [21]. These different aspects were collectively addressed, and we learned through the process. The lessons included the need for rapid and wide-spread internal communications and for excellent flexibility and adaptability to an evolving and ambiguous situation, which are of the utmost importance when accurate evaluations and knowledge are lacking.

Among the clinical activities, surgery was the most rapidly and deeply affected for several reasons. Initially, although not required to directly care for COVID-19 patients, we were asked by regional health authorities to make ICU beds available as much as possible, in case of the predicted overwhelming shortage of ICU capacity in other hospitals. To fulfill this obligation, previously scheduled major surgeries were postponed according to the recommendations of the scientific community and health agencies [16,17,21], while the number of active operating rooms were reduced to free up respirators. Furthermore, many patients, concerned by the possibility of infection, elected to postpone their surgery even if recommendations against this decision were given [25]. Finally, a substantial number of our surgical patients come from referrals by private practice doctors. Because of the lockdown instructions, the majority of these and the corresponding laboratories shut their doors while, at the same time, decreasing the number of new diagnoses. When it became evident that we would not be massively impacted, returning to normal was not easy for multiple reasons: private practices remained closed in accordance with national regulations, while patients were still reluctant to proceed with surgery. In addition, surgeons and anesthesiologists expressed concern for patients and their own safety regarding the large number of asymptomatic or undiagnosed patients.

At the end of the study period, returning to normal activity remained a very distant possibility. We were still collectively working on balancing clinical activity between several opposing realities. Patients still expressed fears of being infected while visiting the center or of major complications if a COVID-19 infection occurred after the administration of cancer treatment. In parallel, nursing and medical staff stated their apprehension with regards to exposure to COVID-19 infection via undiagnosed patients. Clinicians were also highly concerned by the new paradigm of risk-benefit analysis that they had not yet totally mastered; the possible years of life lost by not administering treatment to a patient versus the uncertainty of poorly defined complications due to COVID-19 infections in treated patients [26]. Furthermore, some of our protocol modifications were perturbing; for years we had advocated for more direct human contact with patients and family, and the switch to teleconsultations negatively impacted the relationships we had aimed 
Table 2 Lessons from our COVID-19 experience

\begin{tabular}{|c|c|c|}
\hline Topic & Methods & Personnel \\
\hline $\begin{array}{l}\text { Role of the hospital } \\
\text { management }\end{array}$ & $\begin{array}{l}\text { To define the hospital's general strategy } \\
\text { To set-up and lead a crisis unit } \\
\text { To make and promote decisions to be followed by all staff via information } \\
\text { from crisis unit } \\
\text { To review, and update as needed, standard hospital operating procedures } \\
\text { To assess supplies stockpiled as actually or potentially jeopardized by } \\
\text { crisis reality } \\
\text { To maintain real-time communication with regional and national } \\
\text { health authorities } \\
\text { To manage information: } \\
\text { Rapid dissemination of general and specific information relevant to } \\
\text { hospital staff, patients, and corresponding partners } \\
\text { Organization of bottom-up reporting from hospital personnel } \\
\text { Dissemination of information for creating and using adapted } \\
\text { communication tools: newsletters, social network, hospital } \\
\text { website, hospital app, phone hotline } \\
\text { To reallocate human resources according to needs }\end{array}$ & \\
\hline Crisis unit & $\begin{array}{l}\text { Composition needed to be adapted to the actual crisis: } \\
\text { General direction } \\
\text { Directors/representatives from clinical departments and medical platforms } \\
\text { Directors from logistic platforms } \\
\text { Frequency: } \\
\text { As frequent as needed and by request from the administration } \\
\text { Missions: } \\
\text { To advise hospital administration on all subjects } \\
\text { To hierarchically convey and explain decisions from the administration } \\
\text { To report on the actual situations of each department } \\
\text { To report and prospectively evaluate consequences on each } \\
\text { department's activity } \\
\text { To propose a strategy for staff safety } \\
\text { To propose a strategy for patients' care and safety } \\
\text { To propose innovation in patient care according to crisis characteristics }\end{array}$ & \\
\hline $\begin{array}{l}\text { Patient safety and care } \\
\text { continuation }\end{array}$ & $\begin{array}{l}\text { To implement and respect safety procedures issued by hospital administration } \\
\text { To evaluate best treatment strategy according to each patient's disease, status, } \\
\text { and crisis evolution } \\
\text { To maintain high level communication and support with patients and outside } \\
\text { partners } \\
\text { To use provided psychologic support }\end{array}$ & $\begin{array}{l}\text { Staff and patients } \\
\text { Medical community and patients } \\
\text { Medical community and administration } \\
\text { Patients }\end{array}$ \\
\hline
\end{tabular}

to build. The impacts on both sides of this relationship still need to be evaluated.

We should also emphasize that the predicted "tsunami" never reached our hospital, as it was successfully contained by first-line hospitals. Yet, the prediction led us, at least partially, to deliberately decrease our clinical activity in preparation for it. Retrospectively, this means that this deliberate decrease could have been entirely avoided, and emphasizes the global lack of pandemic preparedness. This is of great importance, as these decisions have not been free of consequences; some treatments were not administered while some diagnoses were not performed in due time. The individual and global loss of years of life related to the pandemic still needs to be assessed [25-28]. Also, institutional economic well-being, key for development, is closely related to clinical activity. What will be the financial impact of the decrease we observed? As illustrated, it appears that the recovery of postponed clinical activity is far from being obvious, and it may be even worse than what we can currently see.

Indeed, the 2 months preceding the outbreak were characterized by an increase of more than $20 \%$ in the IPC's revenue, as compared to the same months of 2019 , making the shortfall related to the pandemic most likely worse. This is a major concern for long-term investment strategies; both questions will have to be addressed in further analyses.

The debate on the lack of preparation of individual institutions or national health services could go on forever, but the lessons we have learned in these dark days [7,29] (Table 2) should most certainly be implemented in practical procedures for rapid future deployment, 
to limit any additional damage to both cancer patients and the institutions that care for them when-not if - the next pandemic occurs.

\section{CONFLICTS OF INTEREST}

The authors declare they have no conflicts of interest.

\section{AUTHORS' CONTRIBUTION}

All authors agreed to participate in this study, and contributed by providing data and reviewing the manuscript.

\section{FUNDING}

No financial support was provided.

\section{ACKNOWLEDGMENTS}

- The authors wish to acknowledge other members of the Institute for their specific and outstanding contributions in the management of our hospital in the current situation: Jean Louis Blache, MD; Jean François Moulin, MD; Jean Marc Schiano, MD; and the head nurses Cristelle Oliveri, Laurence Caymaris, and Farida Braham. Management of different services or logistic departments contributed as well to optimizing our organization: Mr. Emmanuel Naudet, Mrs. Martine Bouyssie, and Mr. Damien Bauzat.

- We also express our appreciation to all staff of the Paoli-Calmettes Institute for their excellent performance in carrying out their work and a special thanks to all hospital staff and those of the scientific campus, Marseille Cancer Research Center (CRCM), who volunteered to be relocated to missions other than their usual ones.

\section{SUPPLEMENTARY MATERIALS}

Supplementary data related to this article can be found at https:// doi.org/10.2991/chi.k.210919.001.

\section{REFERENCES}

[1] Huang C, Wang Y, Li X, Ren L, Zhao J, Hu Y, et al. Clinical features of patients infected with 2019 novel coronavirus in Wuhan, China. Lancet 2020;395;497-506.

[2] Liu W, Tao ZW, Wang L, Yuan ML, Liu K, Zhou L, et al. Analysis of factors associated with disease outcomes in hospitalized patients with 2019 novel coronavirus disease. Chin Med J (Engl) 2020;133;1032-8.

[3] Xu Z, Shi L, Wang Y, Zhang J, Huang L, Zhang C, et al. Pathological findings of COVID-19 associated with acute respiratory distress syndrome. Lancet Respir Med 2020;8;420-2.

[4] Zheng Z, Peng F, Xu B, Zhao J, Liu H, Peng J, et al. Risk factors of critical \& mortal COVID-19 cases: a systematic literature review and meta-analysis. J Infect 2020;81;e16-e25.
[5] Liang W, Guan W, Chen R, Wang W, Li J, Xu K, et al. Cancer patients in SARS-CoV-2 infection: a nationwide analysis in China. Lancet Oncol 2020;21;335-7.

[6] Yu J, Ouyang W, Chua MLK, Xie C. SARS-CoV-2 transmission in patients with cancer at a tertiary care hospital in Wuhan, China. JAMA Oncol 2020;6;1108-10.

[7] Desai A, Sachdeva S, Parekh T, Desai R. COVID-19 and cancer: lessons from a pooled meta-analysis. JCO Glob Oncol 2020;6;557-9.

[8] Dai M, Liu D, Liu M, Zhou F, Li G, Chen Z, et al. Patients with cancer appear more vulnerable to SARS-CoV-2: a multicenter study during the COVID-19 outbreak. Cancer Discov 2020;10;783-91.

[9] Williamson EJ, Walker AJ, Bhaskaran K, Bacon S, Bates C, Morton CE, et al. Factors associated with COVID-19-related death using OpenSAFELY. Nature 2020;584;430-6.

[10] Gupta S, Hayek SS, Wang W, Chan L, Mathews KS, Melamed $\mathrm{ML}$, et al. Factors associated with death in critically ill patients with coronavirus disease 2019 in the US. JAMA Intern Med 2020;180;1436-47.

[11] Kuderer NM, Choueiri TK, Shah DP, Shyr Y, Rubinstein SM, Rivera DR, et al. Clinical impact of COVID-19 on patients with cancer (CCC19): a cohort study. Lancet 2020;395;1907-18.

[12] Malard F, Genthon A, Brissot E, van de Wyngaert Z, Marjanovic Z, Ikhlef S, et al. COVID-19 outcomes in patients with hematologic disease. Bone Marrow Transplant 2020;55;2180-4.

[13] Wang D, Yin Y, Hu C, Liu X, Zhang X, Zhou S, et al. Clinical course and outcome of 107 patients infected with the novel coronavirus, SARS-CoV-2, discharged from two hospitals in Wuhan, China. Crit Care 2020;24;188.

[14] Disis ML. Oncology and COVID-19. JAMA 2020;324;1141-2.

[15] Lee LYW, Cazier JB, Angelis V, Arnold R, Bisht V, Campton NA, et al. COVID-19 mortality in patients with cancer on chemotherapy or other anticancer treatments: a prospective cohort study. Lancet 2020;395;1919-26.

[16] Lauer SA, Grantz KH, Bi Q, Jones FK, Zheng Q, Meredith $\mathrm{HR}$, et al. The incubation period of coronavirus disease 2019 (COVID-19) from publicly reported confirmed cases: estimation and application. Ann Intern Med 2020;172;577-82.

[17] Jazieh AR, Al Hadab A, Al Olayan A, AlHejazi A, Al Safi F, Al Qarni A, et al. Managing oncology services during a major coronavirus outbreak: lessons from the Saudi Arabia experience. JCO Glob Oncol 2020;6;518-24.

[18] You B, Ravaud A, Canivet A, Ganem G, Giraud P, Guimbaud R, et al. The official French guidelines to protect patients with cancer against SARS-CoV-2 infection. Lancet Oncol 2020; $21 ; 619-21$.

[19] The UK Coronavirus Cancer Monitoring Project team. The UK coronavirus cancer monitoring project: protecting patients with cancer in the era of COVID-19. Lancet Oncol 2020; $21 ; 622-4$.

[20] The Lancet Oncology. Safeguarding cancer care in a postCOVID-19 world. Lancet Oncol 2020;21;603.

[21] Willan J, King AJ, Hayes S, Collins GP, Peniket A. Care of haematology patients in a COVID-19 epidemic. Br J Haematol 2020;189;241-3.

[22] Wang D, Hu B, Hu C, Zhu F, Liu X, Zhang J, et al. Clinical characteristics of 138 hospitalized patients with 2019 novel coronavirus-infected pneumonia in Wuhan, China. JAMA 2020;323;1061-9. 
[23] Kutikov A, Weinberg DS, Edelman MJ, Horwitz EM, Uzzo RG, Fisher RI. A war on two fronts: cancer care in the time of COVID-19. Ann Intern Med 2020;172;756-8.

[24] Sahu KK, Siddiqui AD, Cerny J. COVID-19 pandemic and impact on hematopoietic stem cell transplantation. Bone Marrow Transplant 2020;55;2193-5.

[25] Hsiang S, Allen D, Annan-Phan S, Bell K, Bolliger I, Chong T, et al. The effect of large-scale anti-contagion policies on the COVID-19 pandemic. Nature 2020;584;262-7.

[26] Wang X, Ferro EG, Zhou G, Hashimoto D, Bhatt DL. Association between universal masking in a health care system and SARS-CoV-2 positivity among health care workers. JAMA 2020;324;703-4.
[27] Papautsky EL, Hamlish T. Patient-reported treatment delays in breast cancer care during the COVID-19 pandemic. Breast Cancer Res Treat 2020;184;249-54.

[28] Sud A, Torr B, Jones ME, Broggio J, Scott S, Loveday C, et al. Effect of delays in the 2-week-wait cancer referral pathway during the COVID-19 pandemic on cancer survival in the UK: a modelling study. Lancet Oncol 2020;21; 1035-44.

[29] Willan J, King AJ, Djebbari F, Turner GDH, Royston DJ, Pavord $\mathrm{S}$, et al. Assessing the impact of lockdown: fresh challenges for the care of haematology patients in the COVID- 19 pandemic. Br J Haematol 2020;189;e224-e7. 